\title{
A statistical approach for analyzing and modeling multibeam echosounder backscatter, including the influence of high-amplitude scatterers
}

Luciano Fonseca, Xavier Lurton, Ridha Fezzani, Jean-Marie Augustin, and Laurent Berger

Citation: The Journal of the Acoustical Society of America 149, 215 (2021); doi: 10.1121/10.0003045

View online: https://doi.org/10.1121/10.0003045

View Table of Contents: https://asa.scitation.org/toc/jas/149/1

Published by the Acoustical Society of America

\section{ARTICLES YOU MAY BE INTERESTED IN}

Memory-efficient approximate three-dimensional beamforming

The Journal of the Acoustical Society of America 148, 3467 (2020); https://doi.org/10.1121/10.0002852

Beam-time delay domain deconvolved scheme for high-resolution active localization of underwater targets

The Journal of the Acoustical Society of America 148, 3762 (2020); https://doi.org/10.1121/10.0002780

Vertical directionality and spatial coherence of the sound field in glacial bays in Hornsund Fjord

The Journal of the Acoustical Society of America 148, 3849 (2020); https://doi.org/10.1121/10.0002868

Feit's seminal article on radiation of sound by a vibrating plate

The Journal of the Acoustical Society of America 149, R1 (2021); https://doi.org/10.1121/10.0002422

Nonlinear ultrasonic technique for the characterization of microstructure in additive materials

The Journal of the Acoustical Society of America 149, 158 (2021); https://doi.org/10.1121/10.0002960

Acoustic source localization with the angular spectrum approach in continuously stratified media

The Journal of the Acoustical Society of America 148, EL333 (2020); https://doi.org/10.1121/10.0002095

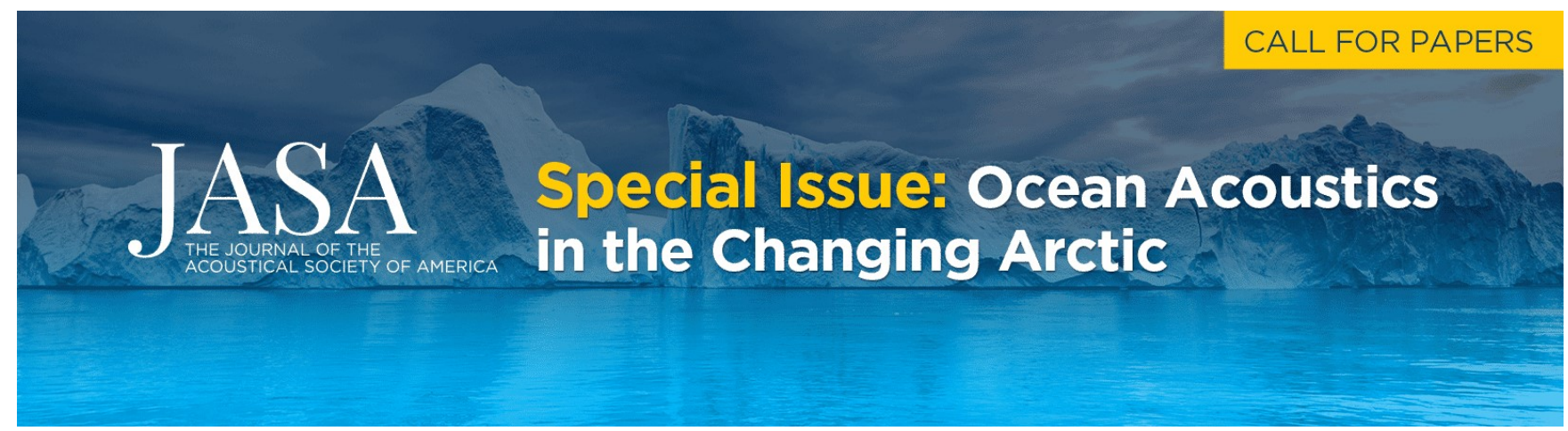




\title{
A statistical approach for analyzing and modeling multibeam echosounder backscatter, including the influence of high-amplitude scatterers
}

\author{
Luciano Fonseca, ${ }^{1, a)}$ Xavier Lurton, ${ }^{2}$ Ridha Fezzani, ${ }^{2}$ Jean-Marie Augustin, ${ }^{2}$ and Laurent Berger ${ }^{2}$ \\ ${ }^{1}$ Department of Electronic Engineering, Universidade de Brasilia (UnB), Brasilia, Brazil \\ ${ }^{2}$ Underwater Acoustic Laboratory, Institut Français de Recherche pour l'Exploitation de la Mer (IFREMER), Plouzané, France
}

\begin{abstract}
:
The statistical analysis of acoustic backscatter samples recorded by multibeam echosounders can be a valuable tool for remote seafloor characterization and interpretation. The present paper aims at analyzing the statistics of backscatter data values, both in "raw" status and after various averaging operations, using field data. It is shown that the statistics of the data can be adequately described by a Weibull distribution parametrized by the incidence angle and the level of applied processing: the distribution of the averaged backscatter amplitude, processed according to various schemes, varies from a Rayleigh law for raw data to lognormal and finally to Gaussian distribution after successive averaging operations. Based on these results, some recommendations for the calculation of the mean backscatter strength are presented. Finally, the influence of high-amplitude scatterers in the backscatter probability density function is addressed; a scheme is suggested to separate the contributions of the substrate from the contributions of the scatterers on the statistical distribution of sonar data samples. (C) 2021 Acoustical Society of America. https://doi.org/10.1121/10.0003045

(Received 19 October 2020; revised 9 December 2020; accepted 11 December 2020; published online 7 January 2021)

[Editor: Nicholas P. Chotiros]

Pages: 215-228
\end{abstract}

\section{INTRODUCTION}

Remote seafloor characterization is a particularly important task for a wide range of applications, including delimitation of benthic habitats and geological seafloor facies or assessment of geotechnical properties, among others (Lamarche and Lurton, 2018). Due to intrinsic difficulties in directly accessing and sampling the seafloor, acoustic methods using sidescan sonars and single-beam and multibeam echosounders (MBESs) are most often employed for underwater surveying. MBESs are the most widely used systems today, scanning the seafloor with a high number of narrow beams across a wide swath across the sonar platform trajectory (Lurton et al., 2015). For each transmitted ping, one time series of backscattered signal is recorded for every acoustic beam formed in reception. These time series are used first for bathymetry measurement, based on their intensity-time dependence or on their interferometric phase characteristics (Lurton, 2010). However, the same time series are also usable for measuring the intensity level of backscatter and hence the acoustical reflectivity of the seafloor after correcting for the sonar sensitivity, propagation losses, and signal footprint extent (Lurton et al., 2015). The so-obtained backscatter strength (BS) is itself related to the seafloor physical characteristics (impedance and roughness) and hence a valuable descriptor for classification and characterization.

\footnotetext{
${ }^{\text {a)} E l e c t r o n i c ~ m a i l: ~ l u c i a n o f o n s e c a @ u n b . b r ~}$
}

Upon acquisition, backscatter time series are recorded at a high sampling frequency, and they can feature a high number of samples, corresponding to a very fine spatial resolution, which may be unnecessary for seafloor mapping applications; moreover, they show a randomly fluctuating character, caused by the very process of backscatter, classically described as echoes from a population of local point targets whose contributions mix with random amplitudes and phases (Stanton et al., 2018). The details of such a distribution of signal amplitude values are not significant at the scale of time samples and should be more usefully considered from a statistical point of view; it is not necessary to use all samples of the time series, and a limited number of parameters describing their distribution is expected to be sufficient.

For practical applications, it is necessary to (1) average the original sample values of the time series-at various steps of acquisition, processing, and analysis of the data-to decrease the noise level and to bring the resolution to a practically usable scale (typically one averaged value per beam is enough for many applications while being consistent with the bathymetry data) and (2) to analyze the statistics of backscatter (before and/or after averaging) to possibly link them to the actual characteristics of the seafloor. Regarding the latter point, at a given acoustical frequency, the backscatter statistical characteristics depend both on the seafloor nature and features and on the incidence angle of the signal acquisition, hence varying across a swath covered in one ping by a MBES (Fezzani et al., 2019). 
MBES manufacturers currently implement such averaging functions in their systems and provide various solutions for the backscatter data conditioning: more often than not, the backscatter samples are pre-filtered and are averaged at various scales before being stored in datagrams; a common process is to provide an average BS value associated with each beam. The situation is even further complicated by the post-processing software suites designed for post-processing of backscatter data, which also propose a number of similar operations of filtering, averaging, and sub-sampling. As a consequence, a large discrepancy prevails in the results obtained by users working with different MBESs and software tools. This has been pointed out by the work of the GeoHab Backscatter Working Group (Lurton et al., 2015) and was the purpose of a dedicated comparison project (Malik et al., 2019).

The present paper aims at analyzing the statistics of BS values from field data recorded by MBESs, both in their "raw" status and after various averaging operations. It will be shown that the statistics of the backscatter amplitudes can be adequately described by a single statistical law, parametrized by both the incidence angle of the backscatter process and the averaging level, and applicable from the raw data to the averaged data (processed according to various schemes).

The statistical process beneath acoustic scattering from the seafloor has been well studied and described in the past. Chotiros et al. (1985) applied autocorrelation function and probability distribution measurements to conclude that the reverberation of a uniform bottom area was consistent with the Rayleigh model. Gensane (1989) observed that the backscattering statistics did not follow a Gaussian distribution but showed an evolution toward a lognormal distribution for longer periods of signal integration. Stanic and Kennedy (1993) reported that reverberation fluctuations measured from a sandy seafloor exhibited large variations of statistical distribution, spreading between Gaussian, lognormal, and Rayleigh distributions. Lyons and Abraham (1999) proposed a statistical characterization of acoustic backscatter at $80 \mathrm{kHz}$, using a mixture of Rayleigh and K-distributions, particularly at high grazing angles. Hellequin et al. (2003) observed that the statistical distribution of echoes from rough seafloors with strong local slope no longer fitted the classical Rayleigh distribution and concluded that the $\mathrm{K}$ distribution proved to be better adapted to the acquired data. Gallaudet and de Moustier (2003) analyzed fluctuations of seafloor, sea surface, and volume acoustic backscatter at $68 \mathrm{kHz}$, applying Rayleigh, K, Weibull, lognormal, and Rayleigh mixture, recommending that Rayleigh mixture models provided the best fits to the backscatter data. Siwabessy et al. (2006) found that either a Rayleigh mixture or lognormal distribution models best fitted the data from four different seabed types, tending to lognormal distributions as the incidence angle increased. Le Chenadec et al. (2007) presented a new model of seabed backscattering statistics, applying a K-distribution to both sidescan and multibeam backscatter. Penrose et al. (2008) noted that fluctuations of the backscatter energy could be satisfactorily modeled by the $\Gamma$-distribution at oblique incidence angles. With respect to these previous works, the present study tries to compose a unifying representation for the statistical law underlying acoustical backscatter, which is applicable to a wide range of incidence angles, to a wide range of frequencies, and to a wide range of processing levels applied to the raw acquired samples. We concentrated a great effort on the calibrations of the transducers, with the aim of acquiring reliable observations. We were also able to include in the proposed statistical distribution the influence of highamplitude scatterers.

\section{BACKSCATTER STATISTICAL ANALYSIS: USING A SIMPLE CASE}

As for any sonar signal, seafloor acoustic backscatter is measured by MBESs under the form of an electrical voltage provided by receiving transducers (hydrophones) sensitive to acoustic pressure. However, BS is intrinsically a measurement of returned energy, hence proportional to the squared acoustic pressure. Therefore, the statistical operations and analyses will more naturally bear on squared amplitudes. The recording and display of backscatter data are most often done in dB-scale, as is generally the case with acoustical data. Considering a set $[x]$ of $N$ samples/observations of backscattered pressure, the fundamental logarithmic expression (in $\mathrm{dB}$ ) of the acoustical level is given by

$$
B S=20 \log _{10} x=10 \log _{10} x^{2}
$$

where BS is in $\mathrm{dB}$ and $x$ is the observed backscatter amplitude (echo acoustical pressure).

This $\mathrm{dB}$ form shown in in Eq. (1) is the usual format of backscatter data recorded by sonar systems. However, for a statistical analysis, it is usually recommended to retrieve the natural intensity values, although directly processing the $\mathrm{dB}$ values can be acceptable under certain conditions.

As an example, we will analyze four study areas in the Bay of Brest: Elorn, Aulne, Rascass, and Renard. These areas were surveyed with a Kongsberg EM2040 MBES at 200, 300, and $400 \mathrm{kHz}$ (Eleftherakis et al., 2018) on Ifremer's $R / V$ Thalia in the framework of the REM2040 annual cruises. Bottom samples and photographs show that the seafloor at Elorn is composed of mud, at Aulne of silty sand with shells, at Rascass of gravelly mud with shells, and at Renard of coarse sand and gravel mixed with coarse elements (Fig. 1).

A compensated backscatter mosaic from a survey line recorded by a Kongsberg EM2040 MBES $(200 \mathrm{kHz})$ in the Bay of Brest, on the Elorn area, is shown in Fig. 2(c). Elorn is an estuary area known to be covered with fine sediments. The acoustic backscatter records were assembled into a compensated backscatter mosaic, following the procedure described by Fonseca and Calder (2005) aimed at correcting the trends in echo level due to propagation losses and backscatter angular dependence. The processing sequence starts with the raw data [Fig. 2(a)] provided by the acquisition 


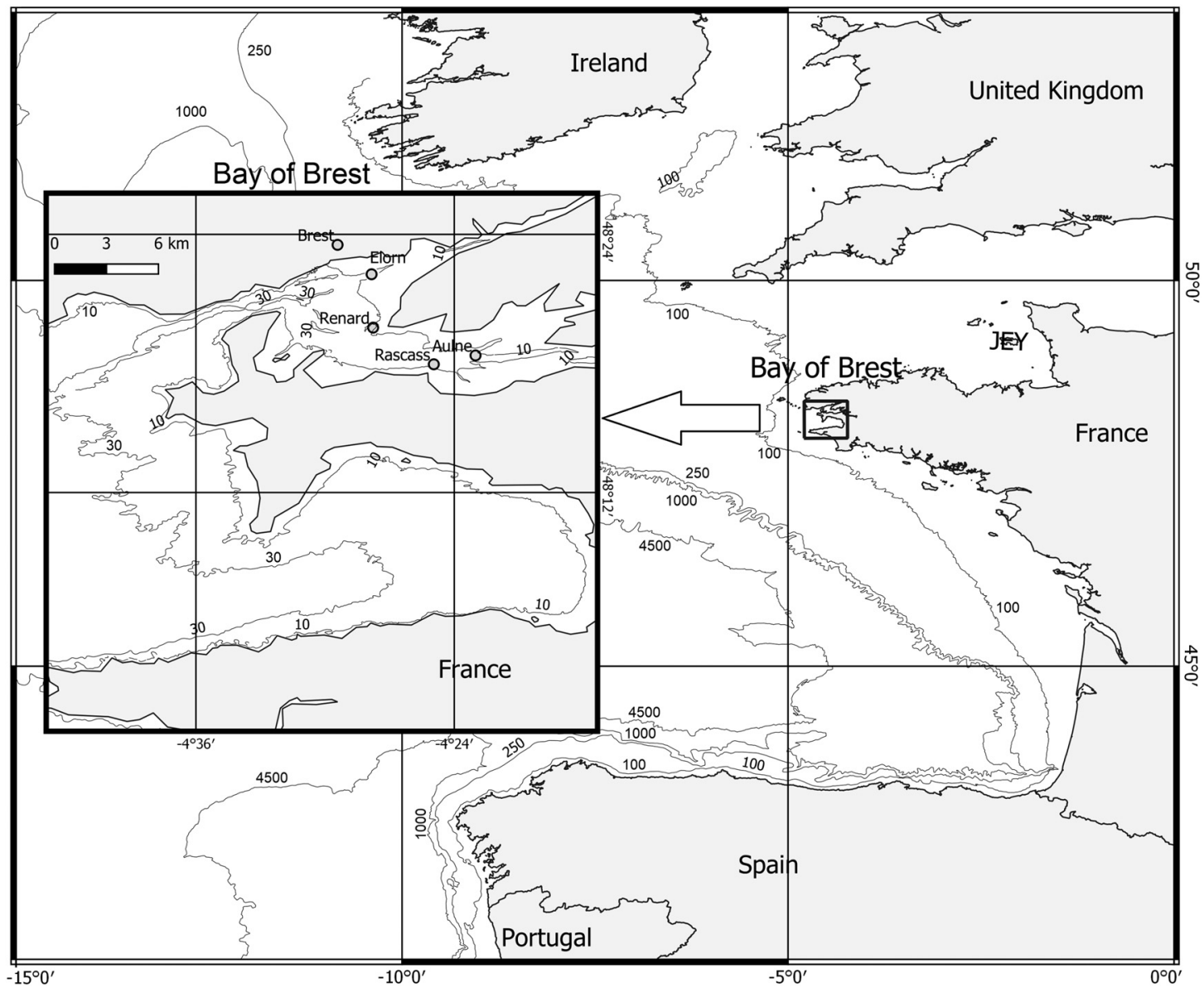

FIG. 1. Location map showing four study areas in the Bay of Brest, Elorn, Aulne, Rascass, and Renard, that were surveyed with a Kongsberg EM2040 MBES at 200, 300, and $400 \mathrm{kHz}$.
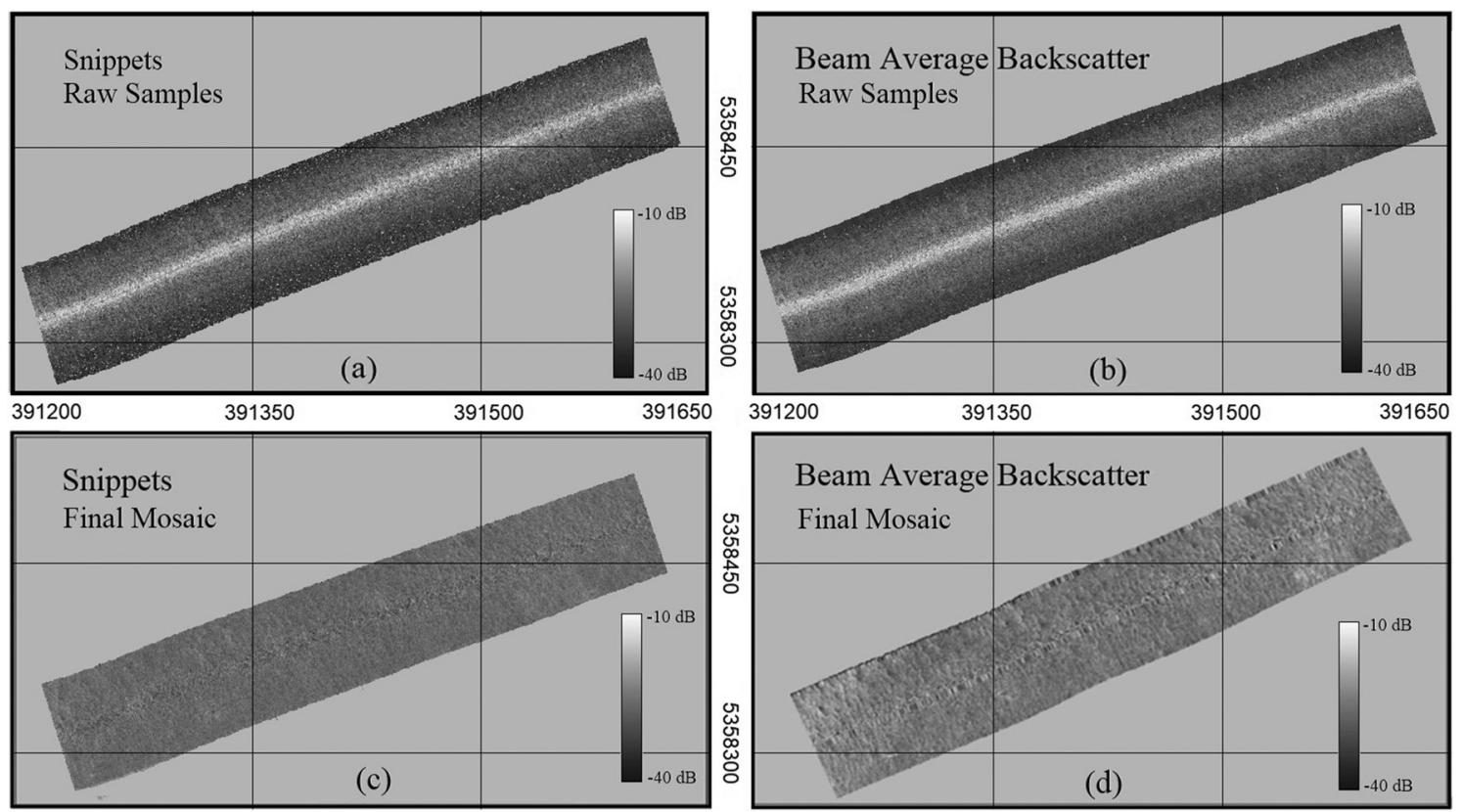

FIG. 2. Seafloor backscatter recorded by a Kongsberg EM2040 MBES ( $200 \mathrm{kHz})$ over a flat and uniform area with sandy mud sediment at $26-\mathrm{m}$ depth, at Elorn area, Bay of Brest, France. (a) Raw time-series samples (snippets). (b) Beam-averaged backscatter samples (one value per beam). (c) Compensated mosaic made from the snippet samples shown in (a), with a mean BS of $0.115(-18.8 \mathrm{~dB})$. (d) Compensated mosaic made from the beam average samples shown in (b), with a mean BS of $0.130(-17.7 \mathrm{~dB})$. The axes' coordinates are in WGS-84 UTM $30 \mathrm{~N}$ projection. 
system. In fact, the sound intensity recorded by the sonar transducer and electronics must be translated into the energy level actually scattered by the seafloor; we call the attempt to apply this transformation "radiometric correction." The BS is conventionally defined per unit of area (Lurton, 2010) so that the actual footprint area of the incident beam should be taken into account for proper radiometric reduction. For this purpose, beam footprint slopes are calculated alongand across-track with respect to a local bathymetric model so that the effective incidence angle for each beam can be calculated. The backscatter angular response is flattened based on a trend removal algorithm, and a residual beam pattern correction is removed on a ping-by-ping basis. Finally, a speckle noise morphological median filter is applied to the data for gap-filling and filtering (Fonseca, 1996). MBES calibration issues (Lurton et al., 2015) are not considered here, and the BS level is arbitrarily (but consistently) scaled. On this scale, the average amplitude of all acoustic backscatter samples in this area is 0.101 , which is equivalent to $-19.9 \mathrm{~dB}$ (also arbitrary reference), and the average BS value, which is the average of the squared amplitude samples [Eq. (1)] is 0.115 (or $-18.8 \mathrm{~dB}$ ).

Looking at the histogram of the distribution of amplitude values [Fig. 3(a)] makes clear that just one mean value of BS $(-18.8 \mathrm{~dB})$ may not be enough to fully characterize the statistical process. In the study cases presented here, it is found that a Rayleigh distribution satisfactorily fits the experimental probability density function (PDF); the adjustment of the PDF to the histogram was done by an annealing optimization (Kirkpatrick et al., 1983). In fact, the amplitude average value of $-19.9 \mathrm{~dB}$ is the mean value of the adjusted Rayleigh distribution. However, the question here remains as to which parameters or which statistics best characterize this statistical process, i.e., the mean, the median, the mode, the decibel mean, the quadratic mean (energy), or other parameters (variance and higher-order moments) of the distribution.

By comparison, if we use one beam-averaged backscatter level per beam [Fig. 2(b)], as obtained from the processing implemented in the MBES (Kongsberg Maritime, 2012), we get a slightly different mosaic [Fig. 2(d)] with a mean backscatter amplitude of 0.122 $(-18.3 \mathrm{~dB})$ and a distribution that is no longer Rayleigh but closer to a lognormal distribution [Fig. 3(b)]. The mean BS value, which is the average of the squared amplitude samples [Eq. (1)], is $0.130(-17.7 \mathrm{~dB})$. Similarly, the presented average amplitude of $-18.3 \mathrm{~dB}$ is the mean value of the fitted lognormal distribution.

It was also observed that backscatter values drawn from final mosaics, for instance values drawn from Fig. 2(c) or 2(d), tend to follow a Gaussian distribution, as shown in the histogram of Fig. 3(c). This is also observed in other processing schemes when the raw backscatter samples are subject to multiple averaging and filtering. This is a direct consequence of the central limit theorem, which states that averaged/filtered samples tend to a Gaussian distribution.
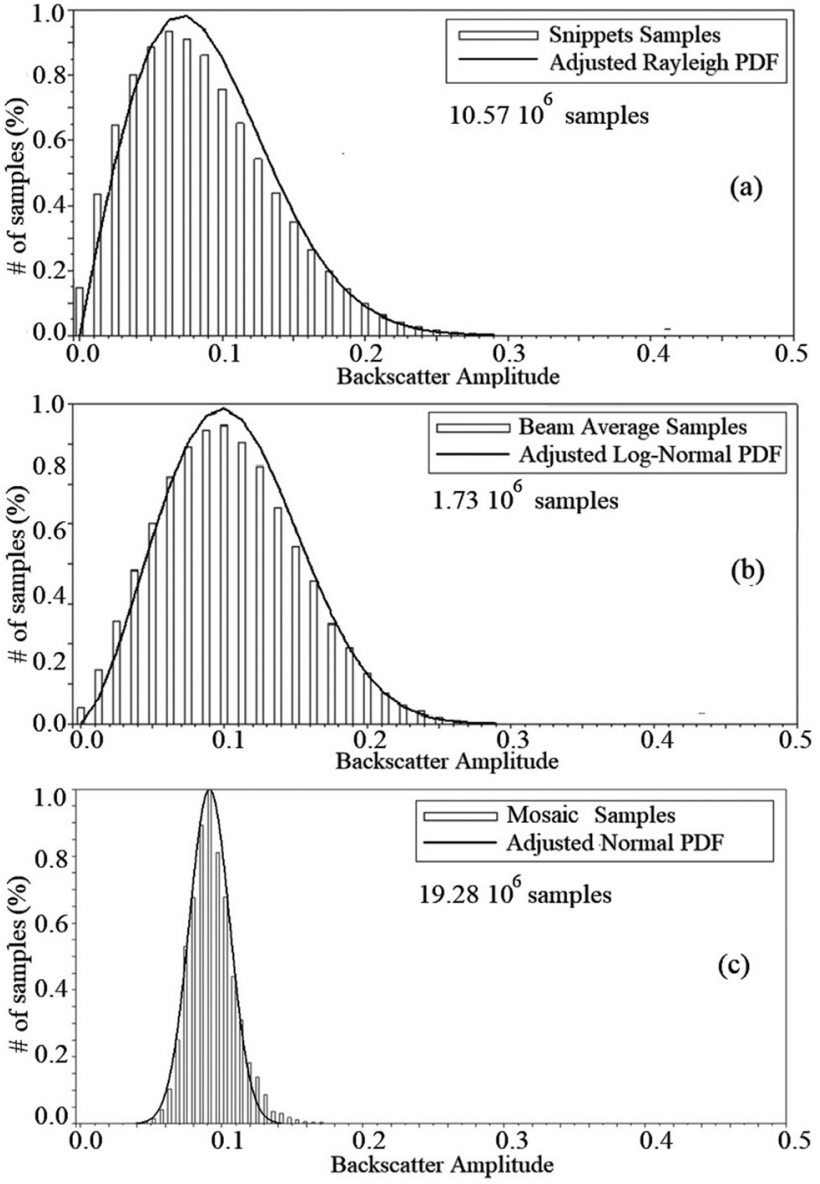

FIG. 3. Illustration of the statistical distribution of seafloor backscatter samples at three stages of MBES processing. (a) Histogram of the raw amplitude values shown in Fig. 2(a) (from the snippets time series per beam). The black continuous curve is the adjusted Rayleigh distribution. (b) Histogram of the raw amplitude values shown in Fig. 2(b) (beam-averaged backscatter). The black continuous curve is the fitted lognormal distribution. (c) Histogram of the mosaic samples of Fig. 2(c). The black continuous curve is the fitted Gaussian distribution.

\section{A. Angular dependence of the backscatter PDF}

A key point in the analysis of seafloor backscatter data is their dependence on the incidence angle. This is illustrated in Fig. 4, which displays distribution histograms of the raw backscatter samples shown in Figs. 2(a) and 2(b). This figure shows that the acoustic backscatter statistics have a strong dependence on the angle of incidence and that the backscatter response at each angle also follows a statistical distribution with its own characteristics. Figures 4(a)-4(c) are the histograms of the raw backscatter amplitudes depicted in Fig. 2(a) at, respectively, low grazing incidence, $45^{\circ}$, and normal incidence. These histograms show that the backscatter amplitudes follow approximately a Rayleigh distribution regardless of the angle of incidence. On the other hand, the histograms on the lower plots [Figs. 4(g)-4(i)] show that the raw beam-averaged backscatter amplitudes from Fig. 2(b) rather follow approximately a lognormal distribution regardless of the angle of incidence. The central plots show the median angular responses (blue line) superimposed on two-dimensional histograms whose color 

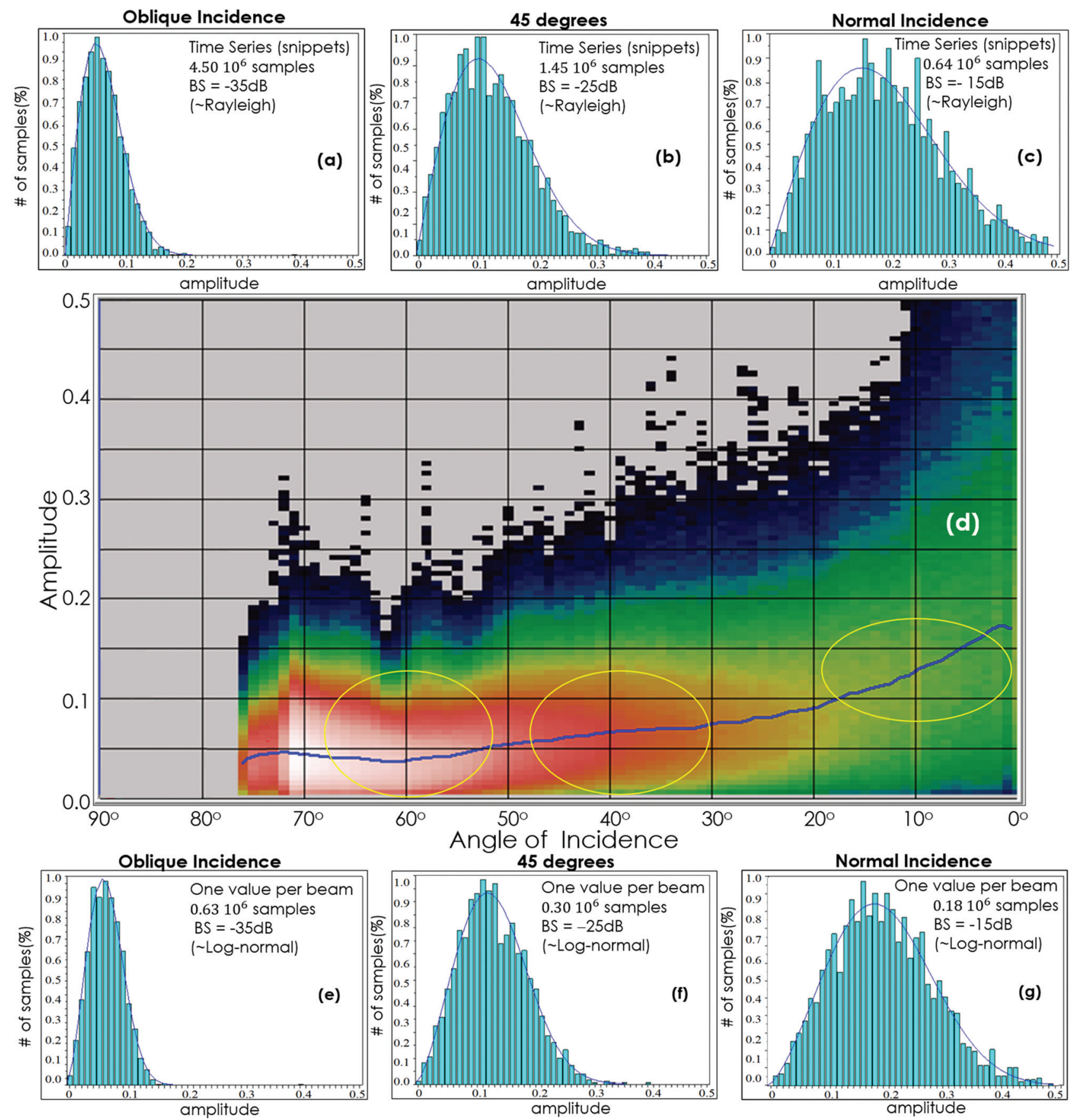

FIG. 4. (a)-(c) Histogram of the raw snippets backscatter samples [Fig. 2(a)] at grazing incidence (a), at $45^{\circ}$ incidence (b), and at normal incidence (c). (d) Median backscatter angular responses of time-series samples shown in Fig. 2(a) superimposed on a 2D histogram, whose color scale reflects the number of samples (from blue/low to red-white/high). (e)-(g) Histogram of the raw beam average backscatter samples [Fig. 2(b)] at grazing incidence (e), at $45^{\circ}$ incidence (f), and at normal incidence (g). Note that the form of underlying statistical distribution usually follows a Rayleigh distribution, in the case of snippets, and moves toward a lognormal distribution, in the case of beam-averaged backscatter, regardless of the acquisition angle.

scale reflects the number of samples (from blue/low to redwhite/high). The 2D histograms show the actual spread of amplitude samples around the median value.

The same behavior of a Rayleigh distribution for time series ("snippets") samples and a lognormal distribution for beam-averaged values was also observed in backscatter samples recorded by a Kongsberg EM710 multibeam sonar $(90 \mathrm{kHz})$ and a Kongsberg EM122 multibeam sonar $(12 \mathrm{kHz})$. Those records are analyzed later in this paper. The observed behavior (from Rayleigh to lognormal to Gaussian) hence appears to be independent of the frequency and independent of the beam incidence angle.

\section{B. Weibull distribution}

The above analysis showed the acoustic backscatter intensities to follow a statistical distribution that depends on the level of processing applied by the acquisition (or post- 
processing) system. The general trend can be summarized as follows: for raw/unprocessed observations, the distribution will be closer to Rayleigh; the higher the processing level, the stronger the averaging, the closer it will be to a Gausslike distribution. Since (as most MBES users) we have actually little control over the processing steps applied during acquisition, we will assume that the backscatter intensities follow a general Weibull cumulative distribution function (CDF) as defined in Eq. (2) (Rinne, 2008). The Weibull distribution has also been successfully used in describing the statistical process observed for radar clutter and electromagnetic scattering (Sekine et al., 1981; Boothe, 1969).

$$
F(x)=1-e^{-(x / \alpha)^{\beta}} ; \quad x \geq 0 .
$$

This CDF is defined by two parameters: $\alpha$, which is related to the central amplitude value, and $\beta$, which is related to the shape of the distribution. If the shape parameter $\beta$ is 1.0 , the distribution is exponential; $\beta=2.0$ corresponds to Rayleigh; $\beta=2.5$ to lognormal; and $\beta=3.6$ to Gaussian. Thus, the Weibull distribution is very flexible and potentially able to fit both time series data (snippets), beam-averaged data, and possibly any other level of processed data. The mean value $\langle x\rangle$, the median value, and the variance of a Weibull distribution are given by Eqs. (3), (4), and (5), respectively,

$$
\begin{aligned}
\langle x\rangle=\alpha \Gamma\left(1+\frac{1}{\beta}\right), & \\
\operatorname{Median}(x)= & \alpha(\ln 2)^{1 / \beta}, \\
\operatorname{Variance}(x) & =\left\langle x^{2}\right\rangle-(\langle x\rangle)^{2} \\
& =\alpha^{2}\left[\Gamma\left(1+\frac{2}{\beta}\right)-\Gamma^{2}\left(1+\frac{1}{\beta}\right)\right],
\end{aligned}
$$

where $\Gamma()$ is the classical gamma function.

\section{Squared-Weibull distribution}

Assuming that a set of backscatter amplitudes $[x]$ follows a Weibull distribution [Eq. (2)], the backscatter intensity $y=x^{2}$ will follow a squared-Weibull distribution. The expression for this new distribution [Eq. (6)], is obtained by replacing $x=\sqrt{y}$ in Eq. (2).

$$
F(y)=1-e^{-(\sqrt{y} / \alpha)^{\beta}} .
$$

The expression for the mean value $\langle y\rangle$ of a squared-Weibull distribution (Eq. 6) can be obtained by substituting Eq. (3) into Eq. (5).

$$
\langle y\rangle=\left\langle x^{2}\right\rangle=\alpha^{2} \Gamma\left(1+\frac{2}{\beta}\right) .
$$

The median value Median(y) of a squared-Weibull distribution, given by Eq. (8), is obtained by making $F(y)=\frac{1}{2}$ in Eq. (6).

$$
\operatorname{Median}(y)=\alpha^{2}(\ln 2)^{2 / \beta}
$$

The offset between $\langle y\rangle$ in decibels $\left(10 \log _{10}\left\langle x^{2}\right\rangle\right)$ and $\langle x\rangle$ in decibels $\left(20 \log _{10} x\right)$ depends only on the $\beta$ parameter and is given by Eq. (9) and plotted in Fig. 5 (dashed-dotted curve). The offset is approximately $1.0 \mathrm{~dB}$ for a Rayleigh distribution $(\beta=2.0)$ and $0.7 \mathrm{~dB}$ for a lognormal distribution $(\beta=2.5)$ and tends to $0.4 \mathrm{~dB}$ for larger values of $\beta$, corresponding to a Gauss-like distribution $(\beta=3.6)$-whatever, once again, the value of $\alpha$.

$$
\begin{aligned}
& 10 \log _{10}\left\langle x^{2}\right\rangle-20 \log _{10}\langle x\rangle \\
& =10 \log _{10} \Gamma\left(1+\frac{2}{\beta}\right)-20 \log _{10} \Gamma\left(1+\frac{1}{\beta}\right) .
\end{aligned}
$$

Similarly, the offset between $\langle y\rangle$ in decibels $\left(10 \log _{10}\left\langle x^{2}\right\rangle\right)$ and the $\operatorname{Median}(x)$ in decibels $\left(20 \log _{10}(\operatorname{Median}(x))\right)$ depends only on the $\beta$ parameter and is given by Eq. (10) and is also plotted in Fig. 5 (dashed curve). The offset is approximately $1.5 \mathrm{~dB}$ for a Rayleigh distribution $(\beta=2.0)$ and $1.0 \mathrm{~dB}$ for a lognormal distribution $(\beta=2.5)$ and tends to $0.4 \mathrm{~dB}$ for larger values of $\beta$, corresponding to a Gausslike distribution $(\beta=3.6)$.

$$
\begin{aligned}
10 & \log _{10}\left\langle x^{2}\right\rangle-20 \log _{10}(\operatorname{Median}(x)) \\
= & 10 \log _{10} \Gamma\left(1+\frac{2}{\beta}\right)-20 \log _{10}(\ln 2)^{1 / \beta} .
\end{aligned}
$$

\section{Log-Weibull distribution}

Normally, the amplitudes recorded by multibeam sonar are given in decibels $\left(z=20 \log _{10} x\right)$. If the average is done in this logarithm space, the $\mathrm{dB}$ samples will follow a logWeibull distribution with CDF (Eq. 11) obtained by replacing $x=10^{z / 20}$ in Eq. (2).

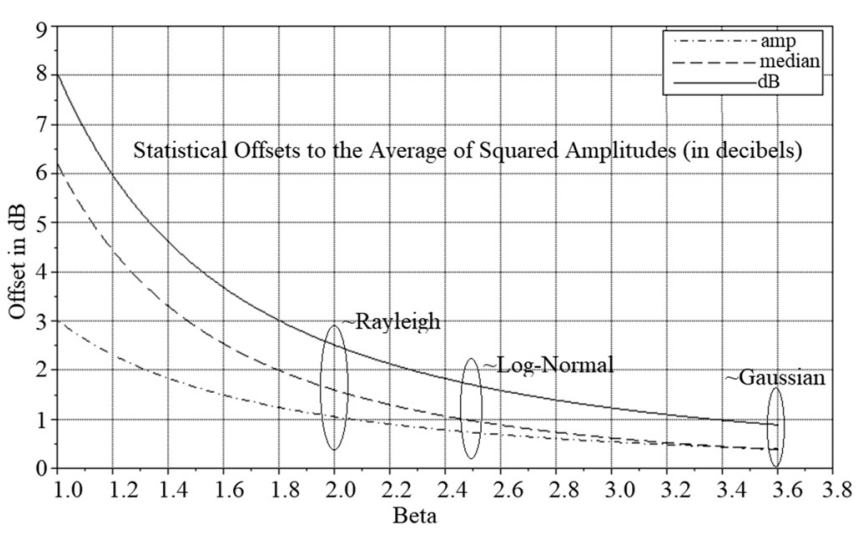

FIG. 5. Statistical offsets (from the direct average of squared amplitudes) calculated for a squared-Weibull distribution related to three different approaches for computing the mean intensity. The offset values (see Table I) depend only on the $\beta$ parameter and are statistically independent of the $\alpha$ parameter. 
TABLE I. Statistical offsets (obtained from Fig. 5) corresponding to the various approaches for computing backscatter averaged levels.

\begin{tabular}{llr}
\hline \hline & $\begin{array}{c}\text { From the average of natural amplitudes } \\
(\# 2)(\mathrm{dB})\end{array}$ & $\begin{array}{c}\text { From the average of dB values } \\
(\# 3)(\mathrm{dB})\end{array}$ \\
\hline Rayleigh distribution (snippets): $\beta=2.0$ & +1.0 & $\begin{array}{c}\text { From the median } \\
(\# 4)(\mathrm{dB})\end{array}$ \\
Log-normal distribution (beam average): $\beta=2.5$ & +0.7 & +2.5 \\
Gaussian distribution (final mosaics): $\beta=3.6$ & +0.4 & +1.7 \\
\hline \hline
\end{tabular}

$$
F(z)=1-e^{-\left(10^{z / 20} / \alpha\right)^{\beta}}
$$

It is not possible to deduce from Eq. (11) an analytical solution to evaluate $\langle z\rangle$, so the moments of this distribution can only be calculated iteratively by means of infinite sum representations (Ortega et al., 2011). As an alternative, we present in this paper results from numerical simulations. First, a large number $N$ of samples are drawn from a uniform distribution in the interval $(0.0,1.0)$, generating a set $[u]$. This set is then mapped to a new set $[x]$ of Weibull-distributed samples, with parameters $\alpha$ and $\beta$, through the transformation shown in Eq. (12). While the mean value $\langle x\rangle$ is directly calculated by Eq. (3) and the mean value $\langle y\rangle$ is directly calculated by Eq. (7), the mean value $\langle z\rangle$ is evaluated numerically by taking the average of the samples $[x]$ in decibels (Eq. 13).

$$
\begin{aligned}
& x=\alpha[-\ln (1-u)]^{1 / \beta} \\
& \langle z\rangle=\left\langle 20 \log _{10} x\right\rangle .
\end{aligned}
$$

The offset between $\langle y\rangle$ in decibels [calculated from Eq. (7)] and $\langle z\rangle$ [obtained from numerical simulations of Eqs. (12) and (13)] can be calculated by Eq. (14). Similar to the offsets presented in Eqs. (9) and (10), Eq. (14) depends only on the $\beta$ parameter. The results are also plotted on Fig. 5 (continuous black curve). This offset is approximately $2.5 \mathrm{~dB}$ for a Rayleigh distribution $(\beta=2.0)$ and $1.9 \mathrm{~dB}$ for a lognormal distribution $(\beta=2.5)$ and tends to $0.9 \mathrm{~dB}$ for larger values of $\beta$, corresponding to a Gauss-like distribution $(\beta=3.6)$. All these offset values are summarized in Table I.

$10 \log _{10}\left\langle x^{2}\right\rangle-\left\langle 20 \log _{10} x\right\rangle=10 \log _{10} \Gamma\left(1+\frac{2}{\beta}\right)-\langle z\rangle$

\section{E. Evaluation of the mean backscatter intensity}

Considering a set $[x]$ of $N$ backscatter amplitude samples, we will now compare and discuss four different approaches for computing the mean intensity:

(1) Direct average of squared amplitudes, $10 \log _{10}\left\langle x^{2}\right\rangle$;

(2) Average of amplitudes plus an offset, $20 \log _{10}\langle x\rangle$ + offset;

(3) Average of $\mathrm{dB}$ values plus an offset, $\left\langle 20 \log _{10}(x)\right\rangle$ + offset;

(4) Median plus an offset, $20 \log _{10}(\operatorname{Median}(x))+$ offset.
Approach \#1 is the mathematically correct form to calculate the mean backscatter intensity, following a squaredWeibull distribution and directly providing the correct mean value. The results from approach \#2 follow a Weibull distribution, and those from approach \#3 follow a log-Weibull distribution. The averages calculated by approaches \#2 and \#3 do not directly provide the correct values, as the calculated averages need to be shifted by statistical offsets (given in Table I) to coincide with the results of approach \#1. For calculating the median with approach \#4, it is not necessary to imply any underlying distribution for the random variable $x$, as the increasing order of a set of amplitude values, a set of $\mathrm{dB}$ values, or a set of square-amplitude values remains the same. As a result, the median will be the same for those three sets. However, the median of a squared-Weibull distribution is also shifted from its mean (Fig. 5).

Determining these offset values is an important step in the process of comparing the results provided by various MBES systems or post-processing software tools possibly using different definitions for the averaging of backscatter level values. A key point addressed in this paper is that most of this variability, such as observed in Malik et al. (2019) could be explained by the different averaging procedures used by the various post-processing software suites. The main values of $\beta$ for processing acoustic backscatter are $\beta=2.0$ (snippets with Rayleigh distribution), $\beta=2.5$ (beam-averaged backscatter with lognormal distribution), and $\beta=3.6$ (gridded/mosaicked backscatter with a Gausslike distribution).

The proposed Weibull PDF was adjusted to the histograms of the Elorn area, shown in Figs. 4(a)-4(c) (snippets at grazing, $45^{\circ}$, and normal incidence). The estimated $\alpha$ parameters reflect the median backscatter intensity, and the estimated $\beta$ parameters are coherent with a distribution that is close to Rayleigh $(\beta=2.0)$ but showing a subtle increase toward the normal incidence $(2.12,1.98$, and 1.93). The same behavior was noticed in Figs. 4(g)-4(i) (beam-averaged backscatter at grazing, $45^{\circ}$, and normal incidence). The estimated $\alpha$ parameters reflect the median backscatter intensity, and the estimated $\beta$ parameters are coherent with a distribution that is close to lognormal $(\beta=2.5)$ but showing a subtle increase toward normal incidence $(2.68,2.62$, and 2.55).

Beyond the particular case (Elorn area) considered above, the Weibull distribution proved to be robust in describing histograms of backscatter amplitudes for various types of seafloor. As an example, we show in Fig. 6 histograms of backscatter samples (gray bars) at $300 \mathrm{kHz}$ from the four study areas shown in Fig. 1. All samples were used 

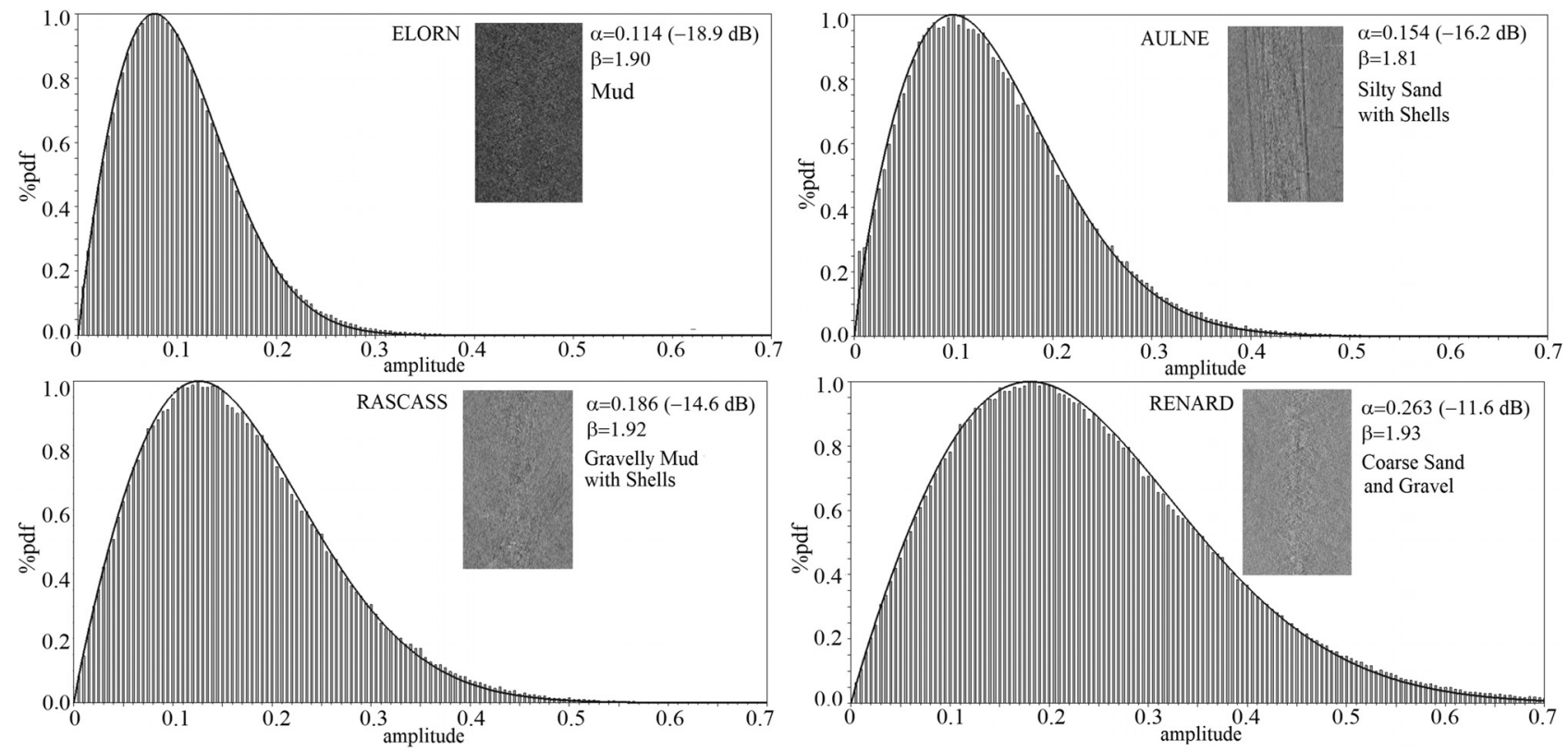

FIG. 6. Histograms of 300-kHz backscatter samples (gray bars) from the four study areas shown in Fig. 1. All samples were used to assemble the histograms, regardless of the incidence angle. The black continuous lines show the adjusted Weibull distribution, with the adjusted $\alpha$ and $\beta$ parameters; the sediment description comes from seafloor samples and photographs. The image insets show the assembled backscatter mosaic for each area (scaled from white/ $0 \mathrm{~dB}$ to black/ $-40 \mathrm{~dB})$.

to assemble the histograms, regardless of their incidence angle. The black continuous lines in Fig. 6 show the adjusted Weibull distribution with the proper offsets; the Weibull $\alpha$ parameter separates perfectly the four areas Elorn (line 292, $\alpha=-18.9 \mathrm{~dB}$ ), Aulne (line 247, $\alpha=-16.2 \mathrm{~dB}$ ), Rascass (line 110, $\alpha=-14.6 \mathrm{~dB}$ ), and Renard (line 049, $\alpha=-11.6 \mathrm{~dB}$ ). Although the four areas show completely different sediment facies on the seafloor, with different mean BS ( $\alpha$ parameter), they all show a similar statistical distribution form close to Rayleigh $(\beta \approx 1.9)$.

\section{MODELING HIGH-AMPLITUDE SCATTERERS}

For a sufficient number of samples recorded over a homogeneous seafloor area and if the required statistical offsets given above are properly applied, all four discussed approaches for calculating the mean BS will give similar results, apart from some statistical fluctuations. However, looking at actual MBES backscatter samples, it is noticed that random noise and high-amplitude scatterers often contaminate the registered values of backscatter amplitudes. Figure 7(a) shows a uniform seabed (a homogeneous sandy substrate), and Fig. 7(b) shows the same sandy substrate with some high-reflectivity scatterers (pebbles). It will be shown that in such situations, these high-amplitude scatterers will completely dominate the averaging of backscatter amplitudes (Goff et al., 2004).

Suppose we acquired acoustic data over the seafloor depicted in Fig. 7(b) and we would like to remotely characterize the seafloor based solely on the acoustic backscatter samples. In a first approach, we would like to characterize the substrate shown in Fig. 7(a), ignoring the highamplitude scatterers. For this purpose, we should definitely avoid averaging together the acoustic samples from the substrate and from the high-amplitude scatterers, as these averaged values from different physical origins would reflect
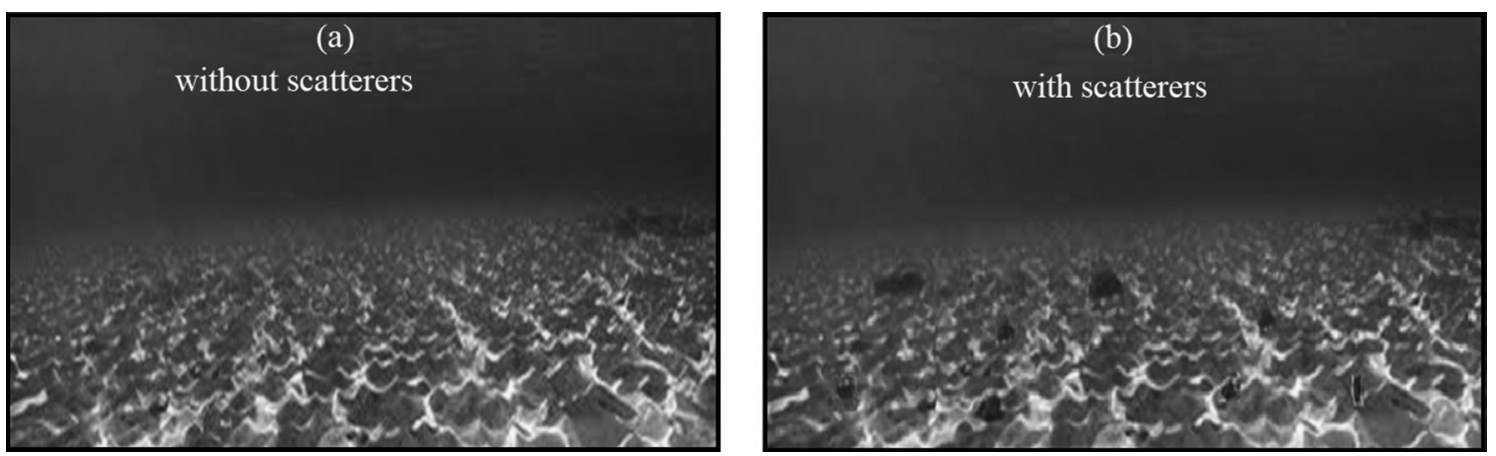

FIG. 7. (a) Underwater photo of a homogeneous sandy seabed. (b) Same sandy substrate with some high-amplitude scatterers. Those scatterers will control the calculation of the mean backscatter intensity. 
neither the substrate nor the scatterers, leading to an erroneous interpretation.

Figure 8 shows a Weibull PDF (black line) characterizing the substrate of Fig. 7(a) with an average level of $-15 \mathrm{~dB}$. In the same figure, we plot a statistical realization of this Weibull PDF (gray), obtained from a numerical simulation. The light gray line shows the same statistical realization (black) plus a random noise at a $10-\mathrm{dB}$ signal-tonoise ratio (SNR). The histogram bars show the statistical realization including noise (light gray curve) together with the contribution of high-intensity scatterers (with average level of $-5 \mathrm{~dB}$ ) affecting $1 \%$ of the backscatter samples. The sonar actually registers the samples corresponding to the histogram bars (substrate plus noise plus scatterers). However, in a remote characterization approach, we would presumably rather like to retrieve the black curve (just the substrate, neither the noise nor the scatterers).

Based on this more realistic statistical modeling (including noise and high-amplitude scatterers), numerical simulations were conducted to determine the most appropriate way to calculate the mean backscatter intensity of the various distributions. Simulations were run for two composite seafloors made of a sediment-like substrate (average BS level, respectively, -15 and $-40 \mathrm{~dB}$ ) and a number of sparse scatterers affecting $1 \%$ of signal samples with an average level of $-5 \mathrm{~dB}$. A random noise was added to all samples with a 10-dB SNR. For both cases, the four averaging approaches were applied, accounting for various numbers of samples and applying the offsets defined in Table I. The results are plotted as a function of the number of samples used in the averaging (from 5 to 100). It can be observed (Fig. 9) that, in the presence of random scatterers and noise, the results of the four averaging approaches differ considerably (up to $20 \mathrm{~dB}$ ), depending on the amount of noise, the number of contributing scatterers, and the number of samples considered in the averaging process.

From these simulations, it can be seen that in the presence of noise and high-amplitude scatterers, direct averaging

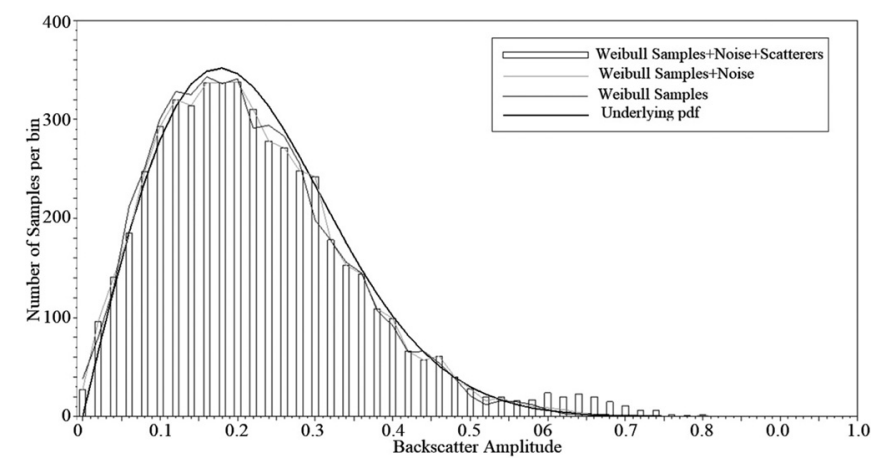

FIG. 8. Histogram of the backscatter amplitude PDF for data recorded over a homogeneous sandy seabed with additive noise and sparse scatterers [Fig. 7 (b)]. The black line shows the underlying Weibull PDF with parameters $[\alpha, \beta]$ simulating the seafloor substrate backscattering without noise and without diffusers. The gray line depicts one statistical realization of the PDF. The light gray line shows the PDF plus a random noise of $10-\mathrm{dB}$ SNR. The histogram bars show the final samples, which include both additive noise and contributions of high-intensity scatterers.

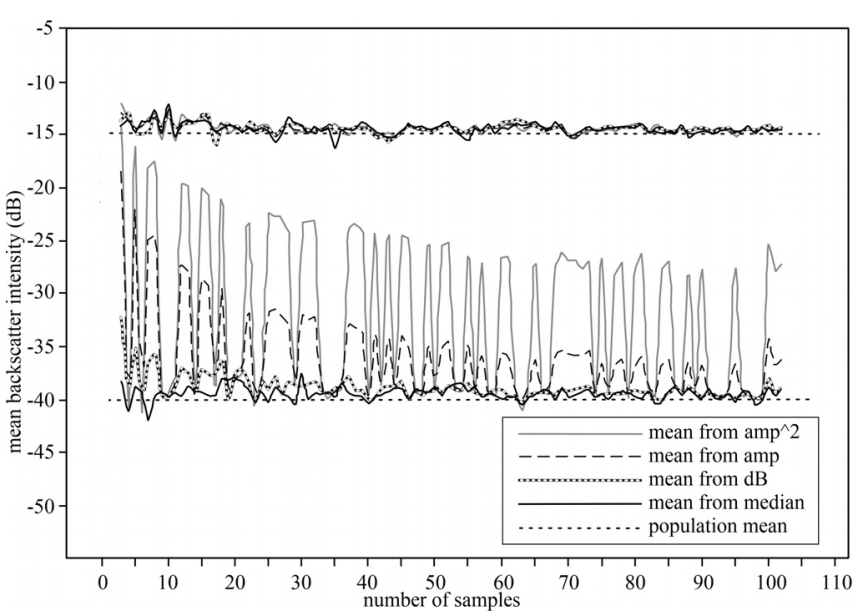

FIG. 9. Calculation results of the mean backscatter intensity as a function of the number of averaged samples for two simulated environments with sediment substrate backscatter levels of -15 and $-40 \mathrm{~dB}$, the addition of $1 \%$ high-intensity (with average level of $-5 \mathrm{~dB}$ ) scatterers, and additive noise (10-dB SNR), following four different calculation methods and appropriate offset compensations (Table I).

of squared amplitudes should be avoided. Although this approach is mathematically correct, its result is severely contaminated by noise and high-amplitude scatterers (continuous gray curve). Figure 9 shows that the estimation errors are maximal for low values of substrate backscatter $(-40 \mathrm{~dB})$ and negligible at high values $(-15 \mathrm{~dB})$. Indeed, the estimation errors increase considerably for lower backscatter values.

Averaging of amplitudes should also be avoided, as it is also severely contaminated by noise and high-amplitude scatterers (dashed black curve). This average does not

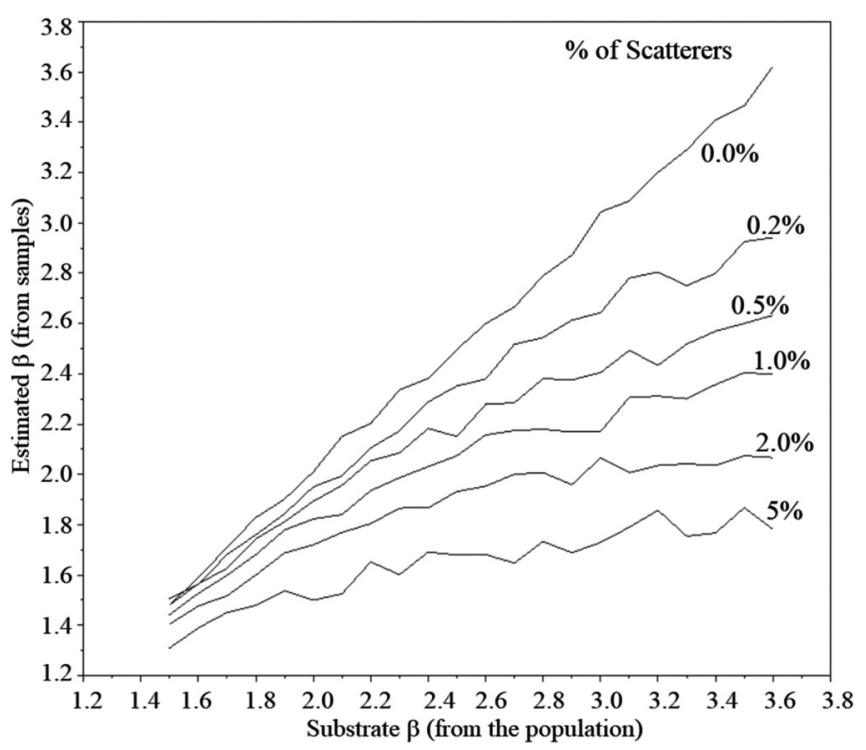

FIG. 10. Correlation between the substrate $\beta$ (from the population) and the estimated $\beta$ (from the samples). This simulation was done for 500 samples and for $\alpha=0.2$. Without scatterers, the estimated $\beta$ agrees with the substrate $\beta(0.0 \%$ line). However, in the presence of scatterers (with average level of $-5 \mathrm{~dB}$ ), the estimated $\beta$ becomes systematically lower that the substrate $\beta$, and the offset appears to be directly proportional to the density of highamplitude scatterers. 


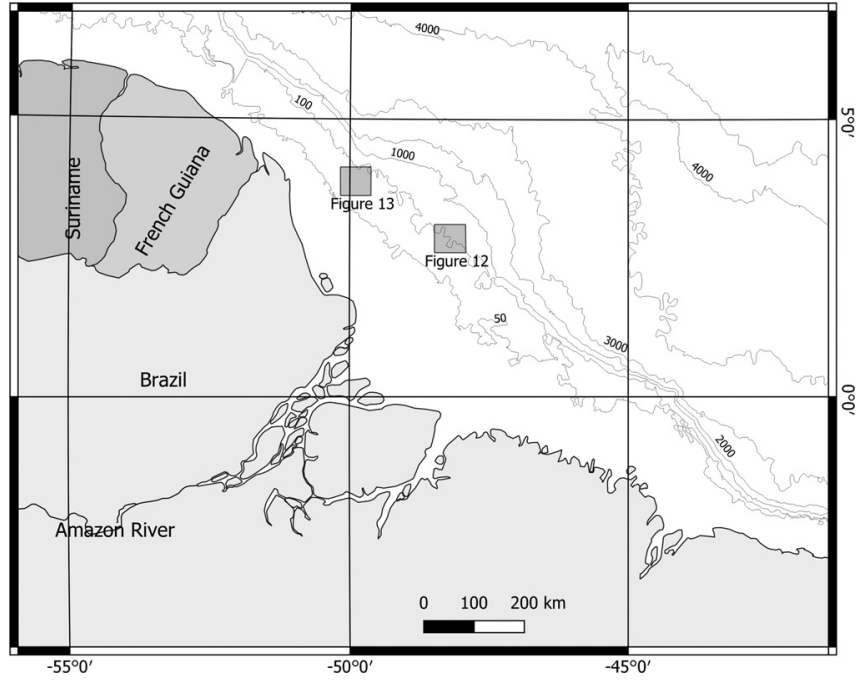

FIG. 11. Map showing the location of the two studied areas at the mouth of the Amazon River, at a water depth of approximately $100 \mathrm{~m}$. The two areas are detailed in Figs. 12 and 13. follow the same PDF as the average of squared amplitudes, so it should be shifted by a statistical offset (Table I). Nevertheless, the actual difference between these two averages is not consistent and is not predictable, depending on the noise level, the number of high-amplitude scatterers, and the underlying distribution. Again, the estimation errors are maximal for low backscatter values.

The average of $\mathrm{dB}$ values does not follow the same PDF as the average of squared amplitudes, so it also needs to be shifted by a statistical offset (Table I). Again, the actual difference between these averages is not consistent and is not predictable, depending on the underlying distribution, the noise level, and the number of scatterers (black and white patterned curve). However, this average is seldom more than $2.5 \mathrm{~dB}$ away from the true value. The estimation errors have a weak dependence on backscatter values.

Finally, the median calculation method (approach \#4) appears to be preferable. The median is a robust measurement weakly affected by the additive noise level, neither by the number of scatterers nor by the number of samples

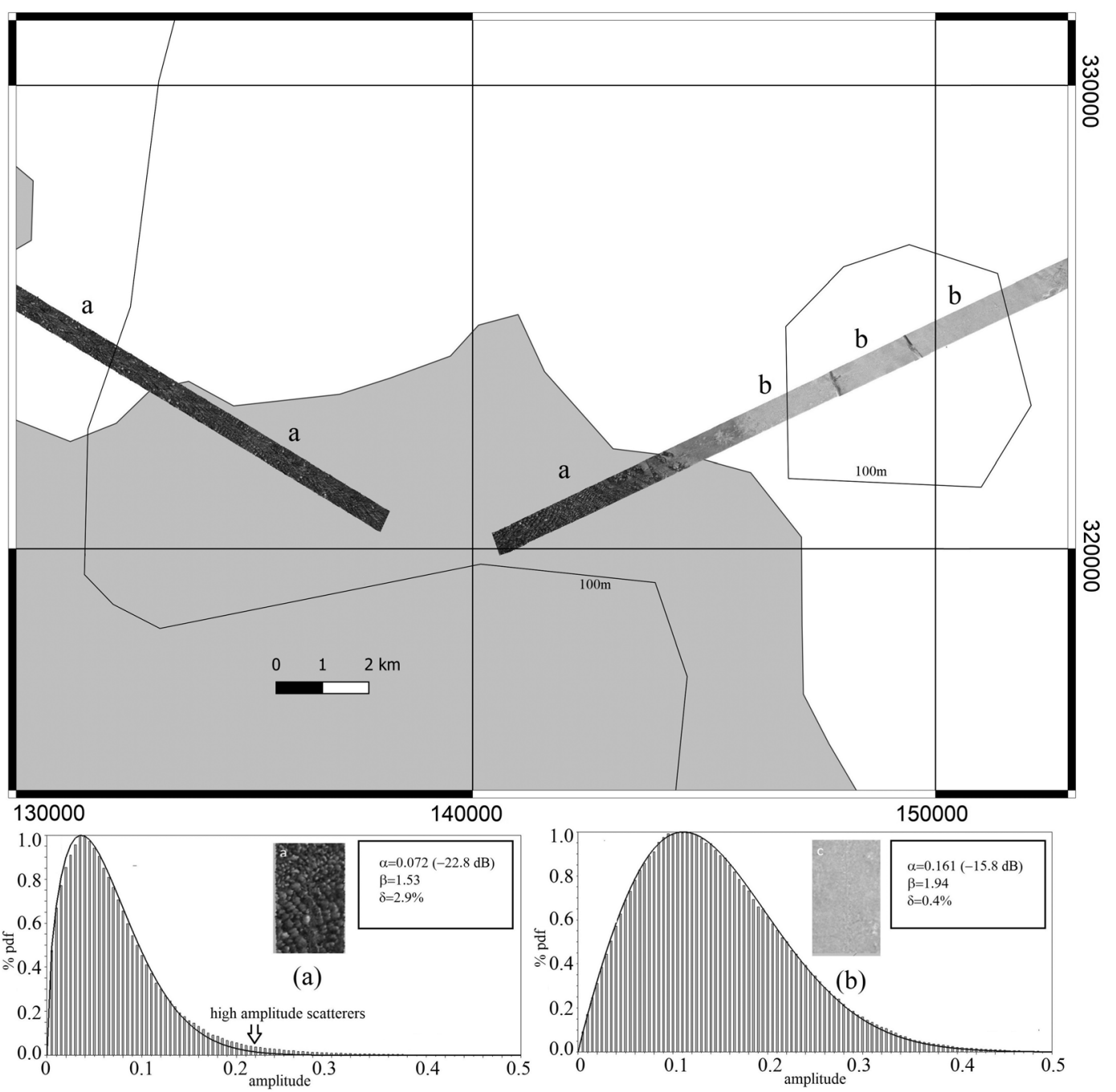

FIG. 12. Analysis of backscatter distributions for data using an EM710 MBES (90 kHz) in an area with interpretation of coral structures either present (a) (gray area) or absent (b) (white area). The graphs on the bottom show the measured histograms (gray bars) and the adjusted probability density distributions (continuous black curves). Scale for backscatter mosaics and insets: from white/0 dB to black/-40 dB. The axes' coordinates are in WGS-84 UTM $23 \mathrm{~N}$ projection. 
(continuous black curve). Similarly, the median result must be shifted by an offset to estimate the correct distribution mean. However, this offset is only dependent on the underlying distribution (Rayleigh for time series samples or lognormal for beam-averaged backscatter; see Table I).

Using the median values indirectly removes the outliers from the observed distributions, in a way that practically excludes the contribution of high-amplitude scatterers from the analysis. The remaining acoustic values will then reflect only the substrate contribution. Once the substrate has been defined, we can compare the relative density of scatterers in adjacent areas by accounting for the difference of the initial distribution and the distribution without the outliers. This technique can be used to detect and quantify the relative number of high-amplitude scatterers (spatial density).

Figure 9 also shows that the median is a very robust estimator for the $\alpha$ parameter. Even with fewer samples, we could still get a reasonable estimation for the central value. On the other hand, the estimation of the $\beta$ parameter is more unstable and demands a large number of samples for a reliable estimation. Even if a large number of samples is available, the estimated $\beta$ will still be biased by the presence of scatterers, as shown in Fig. 10 below. High-amplitude scatterers, even in small amounts, can distort the PDF toward an exponential distribution, with a clear effect of lowering the estimated $\beta$. This observation strengthens the argument that a smaller $\beta$ parameter can be regarded as evidence of the presence of scatterers.

\section{REMOTE CHARACTERIZATION OF HIGH- AMPLITUDE SCATTERERS}

To test the suitability of the analysis of high-amplitude scatterers for the remote characterization of the seafloor, we processed some MBES acquisition lines acquired at the mouth of the Amazon River in the context of the LEPLAC project (Mohriak and Torres, 2017). This project was conducted by the Brazilian Navy with the purpose of establishing the outer limits of the legal Brazilian continental shelf, in accordance with the criteria established by Article 76 of the United Nations Convention on the Law of the Sea (UNCLOS). For this purpose, MBES data were acquired at the Brazilian continental shelf (using a Kongsberg EM710 at $90 \mathrm{kHz}$ ), slope, and rise (using a Kongsberg EM122 at $12 \mathrm{kHz}$, data not addressed here). Figure 11 shows the two areas at the Brazilian continental shelf that were selected for the analysis. These two areas are detailed in Figs. 12 and 13.

Figure 12 shows an area in the mouth of the Amazon River, at a water depth of approximately $100 \mathrm{~m}$ mapped with an EM710 MBES. The acquisition lines cross a sharp change in sedimentary facies, one with rhodoliths [facies (a)] and the other without rhodoliths (b). The gray polygon in this figure delimits an area where coral structures were previously mapped (Amado-Filho et al., 2012; Moura et al., 2012). One histogram was prepared for each area [(a) and (b)] using all backscatter samples registered as beam time series (snippets), restricted to grazing angles from $60^{\circ}$ to $85^{\circ}$, as this angular interval is more sensitive to interface

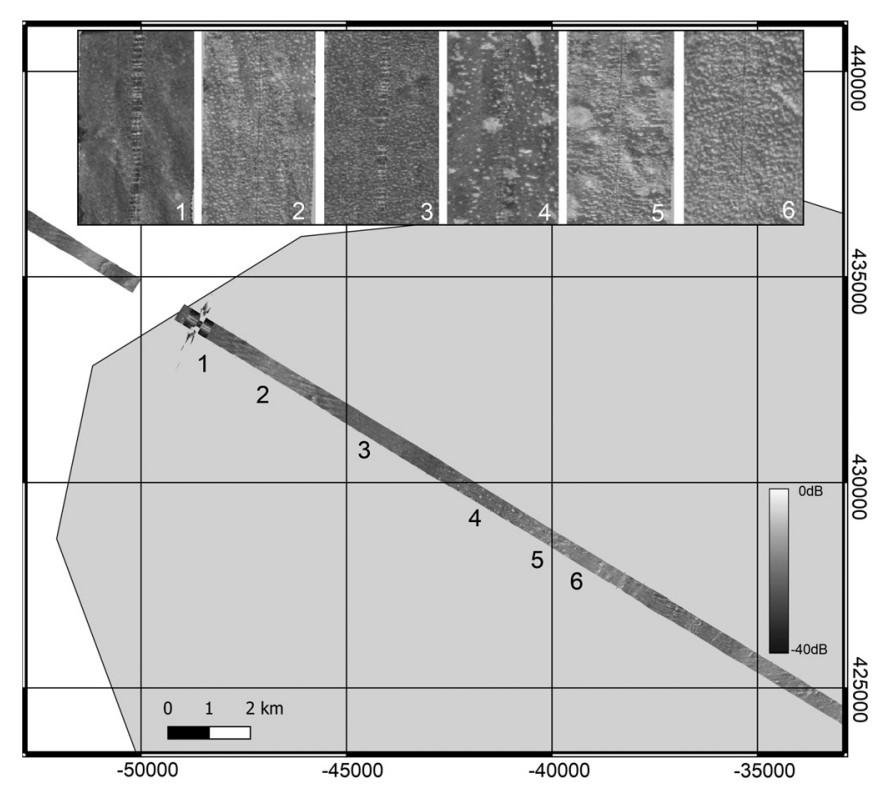

FIG. 13. Acquisition line 168, using a Kongsberg EM710 at $90 \mathrm{kHz}$. Note the ubiquitous presence of rhodoliths along the line, although with different spatial densities and distributions. It is possible to differentiate six distinct seafloor textures (labeled 1-6) along this line. The axes' coordinates are in WGS-84 UTM $23 \mathrm{~N}$ projection.

backscatter. The mean backscatter level of area (b) $(\alpha=-15 \mathrm{~dB})$ is much higher than the value of area (a) $(\alpha=-22.8 \mathrm{~dB})$. On the other hand, the shape of the distribution is closer to Rayleigh $(\beta=1.94)$ in area (b) but shows greater distortion toward an exponential distribution in (a) $(\beta=1.53)$. This distortion is explainable by the presence of high-amplitude scatterers in the histogram, similar to what is shown in the theoretical histogram shown in Fig. 8. For the area (a), note the difference between the measured histogram (gray bars) and the adjusted distribution (black continuous lines) at the highest intensity values (as shown in Fig. 8).

To estimate this difference observed at high-intensity values, we introduce a new parameter $\delta$ defined as the percentual number of samples above the adjusted Weibull distribution (continuous black line in Fig. 8) divided by the total number of samples. For area (a), the $\delta$ parameter is $2.9 \%$, while in area (b), $\delta$ is only $0.4 \%$. The $\delta$ parameter shows a good correlation with the spatial density of high-amplitude scatterers. In this particular case, the high-amplitude scatterers correspond to the presence of rhodoliths.

The gray polygon depicted in Fig. 13, also at the mouth of the Amazon River (location map in Fig. 11), shows an area where sediments with more than $50 \%$ of carbonates were mapped (Amado-Filho et al., 2012; Moura et al., 2012). This area was also surveyed with a Kongsberg EM710 MBES $(90 \mathrm{kHz})$. The acquisition line shows a rich change in texture on the ocean floor. Six sonar images with various textures are detailed at the top of Fig. 13, showing different densities, spacing, and size of the rhodolite beds. The histograms of these textures (Fig. 14) show similar $\alpha$ parameters (approximately $-20 \mathrm{~dB}$ ), in contrast with the different levels of mean backscatter perceived in Fig. 13. The mean backscatter levels 

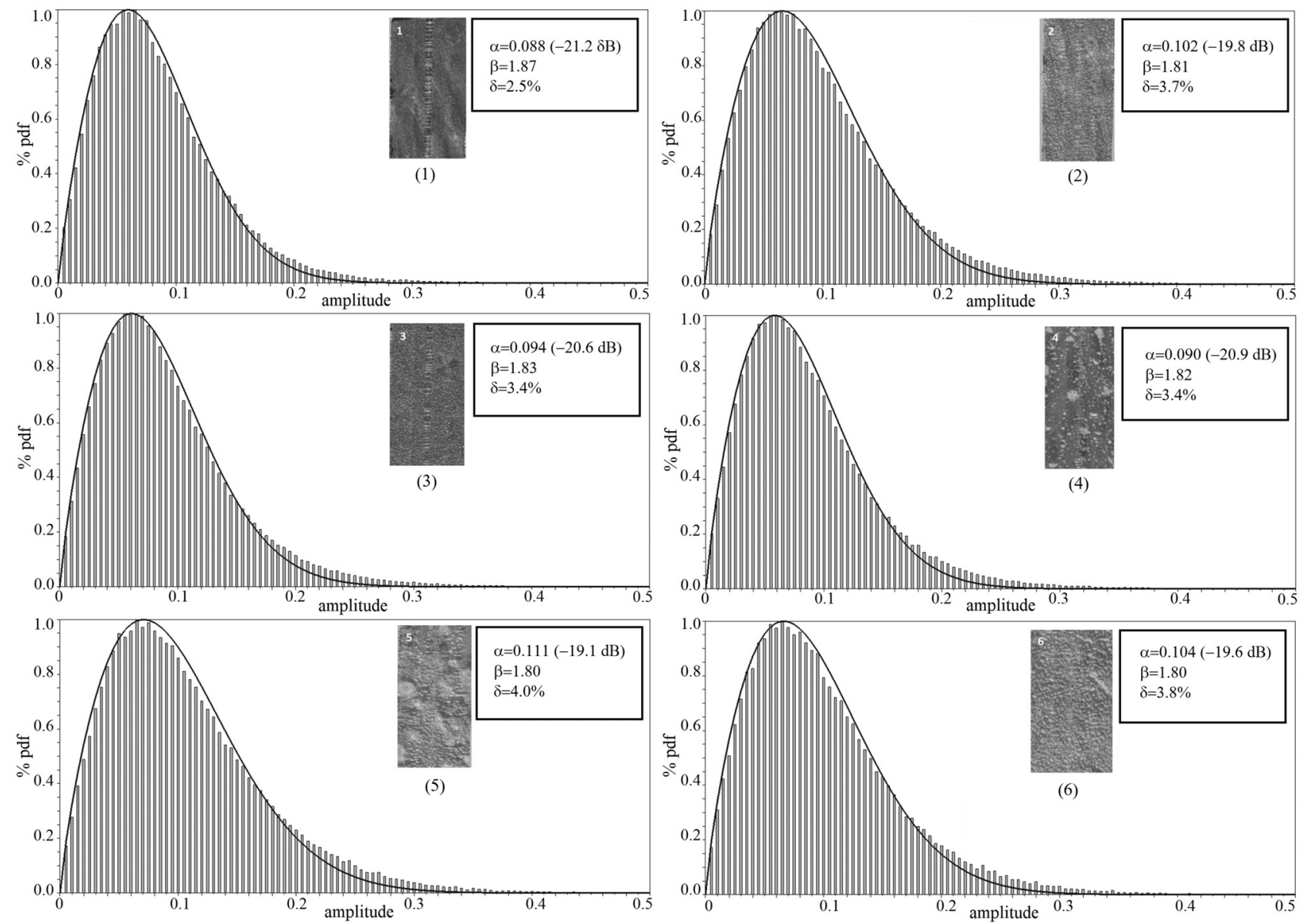

FIG. 14. Statistical analysis of the backscatter values obtained on the six areas defined in Fig. 13. For each area, the gray bars show the measured histogram, and the black continuous lines show the adjusted Weibull PDF. The tables show the model parameters $\alpha$ and $\beta$ of the fitted PDF and the difference $\delta$. Note that the six areas have $\alpha$ parameters close to $-20 \mathrm{~dB}$ and $\beta$ parameters on the order of 1.8 . The percentage differences $\delta$ between the gray bars and black continuous lines for higher amplitude values may be related to the presence and density of high-amplitude scatterers (rhodoliths). The image insets show the assembled backscatter mosaic for each area (scale: from white $/ 0 \mathrm{~dB}$ to black $/-40 \mathrm{~dB}$ ).

perceived in the image insets are a result of a mosaicking procedure that accounts for all acquired backscatter samples in the area, including samples returned from the substrate as well as samples returned from high-amplitude scatterers. Thus, areas with higher density of rhodoliths would show higher levels of mean backscatter. On the other hand, the $\alpha$ parameters appear to be related only to the substrate, which indicates that the six areas could share the same substrate, but with different densities of rhodoliths. The six areas have $\beta$ parameters on the order of 1.8. This deviation of $\beta$ toward values lower than 2.0 is linked to the presence of highamplitude scatterers. Note the consistent difference between the measured histogram (gray bars) and the adjusted probability distribution (continuous back lines) for higher amplitude values. This difference is captured by the $\delta$ parameter varying from $2.5 \%$ to $4 \%$, indicating increasing densities of high-amplitude scatterers (rhodoliths). By analyzing the six insets in Fig. 13, it can be seen that the $\delta$ differences increase while the $\beta$ parameters decrease (for $\beta$ values smaller than 2) and that this behavior could be linked to the increasing spatial density of high-amplitude scatterers, a fact that should be further investigated.

\section{CONCLUSIONS}

Acoustic backscatter recorded by MBESs, if properly processed and interpreted, is an important source of information for remote seafloor characterization. An important step for such analyses is to recognize that the acoustic backscatter is a random variable following an underlying PDF. It was observed that the raw (unprocessed) time series samples follow a Rayleigh distribution; the beam-averaged backscatter values (one mean value per beam) follow a lognormal distribution; and samples resulting from a higher order of processing, including mosaics, tend to follow a Gaussian distribution. This behavior appears to be independent of the sonar frequency (at least in the frequency range considered here, from 90 to $400 \mathrm{kHz}$ ) and to have a weak dependence on the angle of incidence. This paper proposes the use of a Weibull distribution that will adjust amplitude distribution regardless of the level of processing, which is reflected on the $\beta$ shape parameter. A $\beta$ parameter greater than 2 indicates higher levels of data processing, filtering, and averaging; the higher the level of processing, the more the PDF will depart from Rayleigh and will be close to a Gaussian distribution ( $\beta$ value increasing from 2.0 to 3.6). 
At all processing stages, the mean BS is the average of the squared amplitudes. However, the calculating scheme actually used to obtain this mean BS value can lead to inconsistencies. Indeed, some discrepancies reported in the BS calculated with different processing software suites are likely to be explained by the applied calculating scheme. The more consistent and stable way of calculating the mean BS of a set of amplitude values is to get the median value and then correct it for the appropriate statistical offset (Table I). These results are easily usable for practical applications. In this respect, they have been presented as recommendations to users of MBES backscatter data in the framework of the GeoHab specialized working group (Fonseca et al., 2019) to improve the consistency and reliability of the results processed and intercompared by this community.

This paper has also presented a processing scheme that can separate the contributions of the sediment substrate from the contributions of high-amplitude scatterers. The shape of the backscatter PDF is significantly distorted in the presence of high-amplitude scatterers on the seafloor so that the calculated BS values will relate neither to the substrate (lower-amplitude samples) nor to the scatterers (high-amplitude samples). With the proposed method of adjusting a Weibull distribution, the statistical analysis can be used to separate the two contributions and to detect the presence and spatial density of scatterers. The parameter $\alpha$ from the adjusted Weibull distribution relates to the mean backscatter intensity of the substrate, without the scatterers. The parameter $\beta$ relates both to the presence of scatterers and to the level of processing and averaging of the data. A $\beta$ value smaller than 2 indicates that the PDF is changing from Rayleigh distribution toward an exponential distribution, due to the presence of high-amplitude scatterers. Finally, the $\delta$ parameter can conveniently quantify the level of contributions from the scatterers.

\section{ACKNOWLEDGMENTS}

This work was conducted in the framework of the Ifremer R\&D project R403-006 (Underwater Acoustics). The Scientific Board of Ifremer and the University of Brasilia (UnB) are acknowledged for making possible the sabbatical leave of Professor L.F. at Ifremer, resulting in the works presented here. We would also like to thank the Brazilian Diretoria de Hidrografia e Navegação da Marinha - DHN (Directory of Hydrography and Navigation of the Navy) for making available the dataset from the Mouth of the Amazon River.

Amado-Filho, G. M., Pereira-Filho, G. H., Bahia, R. G., Abrantes, D. P., Verasb, P. C., and Matheus, Z. (2012). "Occurrence and distribution of rhodolith beds on the Fernando de Noronha Archipelago of Brazil," Aquat. Bot. 101, 41-45.

Boothe, R. (1969). "The Weibull distribution applied to the ground clutter backscatter coefficient," Technical Report RE-TR-69-15, ASTIA Document AD691109 (U.S. Army Missile Command, Redstone Arsenal, AL).

Chotiros, N. P., Boehme, H., Goldsberry, T. G., Pitt, S. P., Lamb, R. A., Garcia, A. L., and Altenburg, R. A. (1985). "Acoustic backscattering at low grazing angles from the ocean bottom. Part II. Statistical characteristics of bottom backscatter at a shallow water site," J. Acoust. Soc. Am. 77(3), 975-982.

Eleftherakis, D., Berger, L., LeBouffant, N., Pacault, A., Augustin, J. M., and Lurton, X. (2018). "Backscatter calibration of high-frequency multibeam echosounder using a reference single-beam system, on natural seafloor," Mar. Geophys. Res. 39(1-2), 55-73, https://doi.org/10.1007/ s11001-018-9348-5.

Fezzani, R., Berger, L., Le Bouffant, N., Fonseca, L., Augustin, J. M., and Lurton, X. (2019). "Multispectral acoustic angular backscatter measurement and analysis using tilted EK80 wide band transceiver," in Proceedings of Marine Geological and Biological Habitat Mapping (Geohab 2019), May 13-17, Saint Petersburg, Russia.

Fonseca, L. (1996). "Correções Radiométricas dos Dados Sonográficos da Bacia de Campos" ("Radiometric Corrections of the Sonographic Data from the Campos Basin, Brasil"), in Proceedings of the 8th Brazilian Congress of Remote Sensing, April 14-19, 1996, Salvador-BA, Brazil, pp. 527-533.

Fonseca, L., and Calder, B. (2005). "Geocoder: An efficient backscatter map constructor," in Proceedings of the U.S. Hydrographic Conference, March 29-31, 2005, San Diego, CA.

Fonseca, L., Lurton, X., Fezzani, R., Augustin, J.-M., and Berger, L. (2019). "Some practical recommendations for averaging acoustic backscatter strength," in Proceedings of Marine Geological and Biological Habitat Mapping (Geohab 2019), May 13-17, Saint Petersburg, Russia.

Gallaudet, T., and de Moustier, C. (2003). "High-frequency volume and boundary acoustic backscatter fluctuations in shallow water," J. Acoust. Soc. Am. 114(2), 707-725.

Gensane, M. (1989). "A statistical study of acoustic signals backscattered from the sea bottom," IEEE J. Ocean. Eng. 14(1), 84-93.

Goff, J. A., Kraft, B. J., Mayer, L. A., Schock, S. G., Sommerfield, C. K., Olson, H. C., Gulick, S. P. S., and Nordfjord, S. (2004). "Seabed characterization on the New Jersey middle and outer shelf: Correlability and spatial variability of seafloor sediment properties," Mar. Geol. 209, 147-172.

Hellequin, L., Boucher, J. M., and Lurton, X. (2003). "Processing of highfrequency multibeam echo sounder data for seafloor characterization," J. Ocean. Eng. 28(1), 78-89.

Kirkpatrick, S., Gellat, C. D., Jr., and Vecchi, M. P. (1983). “Optimization by simulated annealing," Science 220, 671-690.

Kongsberg Maritime (2012). EM Series Multibeam Echo Sounders: Datagram Formats (Kongsberg Maritime AS, Kongsberg, Norway).

Lamarche, G., and Lurton, X. (2018). "Introduction to the Special Issue 'Seafloor backscatter data from swath mapping echosounders: From technological development to novel applications'," Mar. Geophys. Res. 39(1-2), 1-3, https://doi.org/10.1007/s11001-018-9349-4.

Le Chenadec, G., Boucher, J.-M., and Lurton, X. (2007). “Angular dependence of K-distributed sonar data," IEEE Trans. Geosci. Remote Sens. 45(5), 1224-1235.

Lurton, X. (2010). Introduction to Underwater Acoustics Principles and Applications (Springer, New York).

Lurton, X., and Lamarche, G. (Eds.) (2015). "Backscatter measurements by seafloor-mapping sonars," Guidelines and Recommendations, 200 pp, http:// geohab.org/wp-content/uploads/2014/05/BSWG-REPORT-MAY2015.pdf.

Lyons, A. P., and Abraham, D. A. (1999). "Statistical characterization of high-frequency shallow-water seafloor backscatter," J. Acoust. Soc. Am. 106(3), 1307-1315.

Malik, M., Masetti, G., Schimel, A. C. G., Roche, M., Dolan, M., and Le Deunf, J. (2019). "Preliminary evaluation of multibeam backscatter consistency through comparison of intermediate processing results," Proceedings of U.S. Hydro 2019, March 18-21, Biloxi, MS.

Mohriak, W. U., and Torres, L. C. (2017). "Levantamentos geofísicos para a delimitação da margem continental brasileira" ("Geophysical Surveys for the Delimitation of the Brazilian Continental Margin"), Revista USP 113, 59-80.

Moura, R. L., Amado-Filho, G. M., Moraes, F. C., Brasileiro, P. S., Salomon, P. S., Mahiques, M. M., Bastos, A. C., Almeida, Marcelo G., Silva Jr., Jomar M., Araujo, B. F., Brito, F. P., Rangel, T. P., Oliveira, B. C. V., Bahia, R. G., Paranhos, R. P., Dias, R. J. S., Siegle, E., Figueiredo Jr., A. G., Pereira, R. C., Leal, C. V., Hajdu, E., Asp, N. E., Gregoracci, G. B., Neumann-Leitão, S., Yager, P. L., Francini-Filho, R. B., Fróes, A., Campeão, M., Silva, B. S., Moreira, A. P. B., Oliveira, L., Soares, A. C., 
Araujo, L., Oliveira, N. L., Teixeira, J. B., Valle, R. A. B., Thompson, C. C., Rezende, C. E., and Thompson, F. L. (2016). "An extensive reef system at the Amazon River mouth," Marine Ecosystems, Sci. Adv. 2, e1501252.

Ortega, E. M. M., Cordeiro, G. M., and Carrasco, J. M. F. (2011). "The loggeneralized modified Weibull regression model,” Braz. J. Probab. Stat. 25, 64-89.

Penrose, J. D., Gavrilov, A., and Parnum, I. M. (2008). "Statistics of seafloor backscatter measured with multibeam sonar systems," Proceedings of the Acoustics '08, 29 June-4 July 2008, Paris, France, pp. 8987-9002.

Rinne, H. (2008). The Weibull Distribution: A Handbook (CRC, Boca Raton, FL).
Sekine, M., Ohtani, S., Musha, T., Irabu, T., Kiuchi, E., Hagisawa, T., and Tomita, Y. (1981). "Weibull-distributed ground clutter," IEEE Trans. Aerosp. Electron. Syst. AES-17(6), 596-598.

Siwabessy, P. J., Gavrilov, A. N., Duncan, A. J., and Parnum, I. M. (2006). "Statistical analysis of high-frequency multibeam backscatter data in shallow water," in Proceedings of the First Australasian Acoustical Societies' Conference 2006, ACOUSTICS 2006: Noise of Progress, New Zealand.

Stanic, S., and Kennedy, E. G. (1993). "Reverberation fluctuations from a smooth seafloor," IEEE J. Oceanic Eng. 18(2), 95-99.

Stanton, T. K., Lee, W. J., and Baik, K. (2018). "Echo statistics associated with discrete scatterers: A tutorial on physics-based methods," J. Acoust. Soc. Am. 144, 3124. 